\title{
ATAS Acupuncture Reduces Chemotherapy Induced Fatigue in Breast Cancer Through Regulating ADROAI Expression: A Randomized Sham-Controlled Pilot Trial
}

This article was published in the following Dove Press journal: OncoTargets and Therapy

\author{
Yun-Fen Li $\mathbb{D}^{1, *}$ \\ De-Quan Liu',* \\ Jian-Yun $\mathrm{Nie}^{l, *}$ \\ De-Dian Chen $\mathbb{D}^{1, *}$ \\ Mei Yan' \\ Zhen Zuo ${ }^{2}$ \\ Liang-Xian Liu ${ }^{3}$ \\ Wei-Yu Wang ${ }^{4}$ \\ Mian-Sheng Zhu ${ }^{2,5}$ \\ Wen-Hui $\mathrm{Li}^{\prime}$
}

'The Third Affiliated Hospital of Kunming Medical University/Yunnan Cancer Hospital, Breast Cancer Surgery Department, Kunming, People's Republic of China; ${ }^{2}$ Yunnan University of Traditional Chinese Medicine, College of Acupuncture, Kunming, People's Republic of China; ${ }^{3}$ Kunming Hospital of Traditional Chinese Medicine/The Third Affiliated Hospital of Yunnan University Traditional Chinese Medicine, Acupuncture Department, Kunming, People's Republic of China; ${ }^{4}$ Servbus Technology (Beijing) Co., Ltd., Technical Service Department, Beijing, People's Republic of China; ${ }^{5}$ ARIATAS, Association Pour la Recherche et I'Information de l'Acupuncture TimeAcupoints-Space, College of Acupuncture, Paris, France

*These authors contributed equally to this work
Objective: To investigate the feasibility and effectiveness of ATAS acupuncture (AcupointsTime-Space Acupuncture) as a non-pharmacological intervention to prevent or relieve chemotherapy-induced fatigue in breast cancer patients undergoing taxane chemotherapy.

Methods: A pilot study in Kunming center with the aim of evaluating 40 patients randomized to 3 groups: ATAS, sham and non-acupuncture with an unequal randomization of 2:1:1. Participants with stage I-III breast cancer were scheduled to receive adjuvant EC4P4 chemotherapy. Participants in the ATAS and sham acupuncture arms received 20 sessions of acupuncture over 20 weeks, non-acupuncture arm received usual care. Evaluation scales, including VAS-F, MFI-20, HDAS, ISI, and blood samples were collected at four timepoints (T1-T4). mRNA sequencing was performed to detect the mechanism of acupuncture.

Results: A total of 581 sessions of acupuncture were performed on patients in the acupuncture group. There was no difference between the three groups in terms of clinical characteristics. Patients randomized to ATAS acupuncture had improved symptoms including fatigue, anxiety and insomnia during the whole process of chemotherapy compared with the other two groups. The VAS-F score of ATAS acupuncture group was decreased compared with non-acupuncture group ( $\mathrm{P}=0.004)$. The score of MFI-20 in ATAS acupuncture group was kept at low level, while the other two groups' scores kept climbing during chemotherapy $(\mathrm{P}=0.016 ; \mathrm{P}=0.028$, respectively). The mechanism of ATAS acupuncture which reduced fatigue and depression may be related to ADROA1, by regulating cGMP/PKG pathway.

Conclusion: This pilot study has demonstrated that ATAS acupuncture can significantly reduce fatigue induced by chemotherapy.

Trial Registration: Chinese Clinical Trials Registry, ChiCTR-IPR-17,013,652, registered Dec 3, 2017. http://www.chictr.org.cn/.

Protocol Version: Version 3.2 dated from 2018/04/20.

Keywords: early stage breast cancer, adjuvant chemotherapy, chemotherapy induced fatigue, ATAS

\section{Statement of Translational Relevance}

Some studies showed that acupuncture can regulate the immune state by adjusting hypothalamus pituitary adrenal axis (HPA axis) and inflammatory factors, alleviating the adverse effects of chemotherapy. However, the effect and mechanism by which acupuncture decreases fatigue is unknown. Our clinical study found: (1) the fatigue and depression scores of breast cancer patients treated with ATAS
Correspondence: Mian-Sheng Zhu;

Wen-Hui Li

Email miansheng@wanadoo.fr;

wenhuili20I4@I63.com
OncoTargets and Therapy 2020:13 ||743-1|754

$1 / 743$

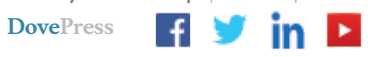

http: $/ /$ doi. org $/ 10.214$ tho 
acupuncture during EC-T chemotherapy were lower than those of the patients treated with only EC-T chemotherapy; (2) the high-throughput sequencing of 146 blood samples at different timepoints from participants found that the expression of ADORA1 and the activity of cGMP/PKG signaling pathway were increased in the ATAS acupuncture group. The studies indicated that acupuncture could reduce fatigue during chemotherapy, the possible mechanism being activation of ADORA1 and regulation of cGMP/PKG pathway, thus down regulating inflammatory factors, regulating HPA axis and playing a role in alleviating depression.

\section{Background}

Fatigue is a major concern in patients with cancer and is estimated to occur in $50-90 \%$ of all cancer patients. ${ }^{1}$ Previous studies showed that 30 to $40 \%$ of breast cancer patients have chronic fatigue after completing chemotherapy, which is higher than comparable populations without prior chemotherapy. ${ }^{2}$ A recent study also showed that $31 \%$ of breast cancer patients are fatigued at the end of chemotherapy. ${ }^{3}$ Post-chemotherapy and/or radiotherapy chronic fatigue has a major impact on the quality of family and society care for cancer survivors. ${ }^{4}$

Unfortunately, we do not have effective pharmacological interventions yet. In recent years, an increasing number of studies of complementary medicine and alternative medicine have been performed. Traditional Chinese medicine has been gradually acknowledged worldwide for the management of cancer related fatigue. ${ }^{5}$ The first publication on the use of acupuncture in cancer related fatigue is dated $2004 .{ }^{6}$ Since then, acupuncture has received attention from researchers as an alternative strategy for cancer-related treatments. Some studies have found that acupuncture can alleviate fatigue and improve life quality when patients finished their treatments. ${ }^{7-12}$ ATAS acupuncture, different from other classical regimens, concerns personalized therapeutical interventions according to the time, the disease and the traditional Chinese medicine diagnosis of patients.

We conducted a randomized sham operation controlled trial to study ATAS as a nonpharmacological intervention to prevent or reduce fatigue in women with early stage breast cancer undergoing adjuvant EC-T chemotherapy weekly for 20 cycles.

\section{Methods \\ Objectives}

The primary aim of the internal pilot was to assess the fatigue in different groups during chemotherapy.

i. To confirm the recruitment feasibility, the retention and assess the compliance to acupuncture appointments;

ii. longitudinal assessments of fatigue through VAS-F scale $^{31}$ weekly during the whole course of chemotherapy and until 1 month after completion of chemotherapy;

iii. self-reported fatigue at T1, T2, T3 and T4. Multiple fatigue inde $\mathrm{x}^{32}$ (MFI-20) consists of 20 items.

Secondary objectives were:

i. self-rated anxiety and depression at T1, T2, T3 and T4. Hospital Anxiety and Depression Scale ${ }^{33}$ (HADS) consists of 14 items.

ii. Sleep quality at T1, T2, T3, T4 assessed by the Insomnia Severity $\operatorname{Index}^{34}$ (ISI).

iii. To find the possible mechanism of reducing fatigue.

\section{Study Design}

The design of the pilot and the main was intended to be identical in all aspects, such as regimens of adjuvant chemotherapy, ATAS acupuncture and Sham acupuncture, and in the method of evaluation of outcomes (measure instruments and time points), except some detailed aspects that needed to be adjusted.

Subjects of the intention to treat population were defined as those who had signed a consent form and completed the baseline questionnaire. The effect of acupuncture on fatigue would be evaluated according to the intention to treat principle (primary analyses) and on a perprotocol basis excluding participants of the acupuncture groups who completed less than $75 \%$ of acupuncture sessions.

A cloud related eCRF (electronic clinical report form) and smartphone platform were established for data entry. In this pilot clinical trial, enrolled patients were randomized into 3 groups: ATAS, Sham and no-acupuncture with an unequal randomization of $2: 1: 1$. The patients were randomized to the true or sham acupuncture group. Next, the acupuncturist logged into the eCRF system to retrieve the assignment. The acupuncturist was the only researcher in the study team who was unblinded to the group assignments.

To maintain the blindness of the study, the data on the true or sham point prescription administered were entered into a separate locked database that only study acupuncturists 
could access. The randomization between acupuncture or noacupuncture was opened for medical team and patients.

\section{Selection of Patients}

Patients aged $>18$ years with a history of stage I to III breast cancer scheduled to receive adjuvant chemotherapy of 4 cycles of EC followed by 4 cycles of Paclitaxel as standard protocol. Trial participants were identified and recruited by clinical and research staff.

\section{Inclusion Criteria}

Voluntary written consent was mandatory before any study related procedure not part of standard medical care took place, with the understanding that the patient may withdraw consent at any time without prejudice to future medical care.

Patients aged $>18$ years.

Recently diagnosed and operated on for primary breast cancer, no evidence of distant metastases.

Patients eligible for adjuvant chemotherapy.

KPS $>60$.

Patients had to be able to comply with the study visit schedule and other protocol requirements.

Patients affiliated with an appropriate social security system.

\section{Exclusion Criteria}

Known severe inflammatory or metabolic disease or any other uncontrolled medical condition or comorbidity that might interfere with subject's participation.

Received acupuncture treatment in the past 4 months.

Received cancer treatment in the five years prior to recruitment (except for basal skin cancer).

Mental disorders, no capacity for civil conduct or with limited capacity for civil conduct.

Needle phobic patients.

Dermatological disease within acupuncture needling area.

\section{Treatment}

\section{Adjuvant Chemotherapy}

The adjuvant chemotherapy performed in Kunming center was a regimen of Epirubicin and Cyclophosphamide followed by Paclitaxel (Epirubicin $90 \mathrm{mg} / \mathrm{m} 2$ plus Cyclophosphamide $600 \mathrm{mg} / \mathrm{m} 2$, q3w x4, followed by Paclitaxel $175 \mathrm{mg} / \mathrm{m} 2$, q2w x4).

\section{Acupuncture}

The patients included in this study received one acupuncture treatment (ATAS or sham acupuncture) weekly, during the whole duration of adjuvant chemotherapy. When applicable, acupuncture was performed on the planned day of chemotherapy for weekly chemotherapy cycles or on day of chemotherapy and in between intervals (day 8 , day 15 , and day 21 for monthly cycles). When acupuncture and chemotherapy took place on the same day, the acupuncture was performed before chemotherapy. Each participant in acupuncture group was supposed to have 20 acupuncture sessions. As long as the participant had more than 15 acupuncture sessions, the case can be analyzed.

Patients in the non-acupuncture arm participated in the evaluations for the full duration of their chemotherapy. Protocol - both the true and sham acupuncture treatments consisted of 60-minute sessions administered weekly including the steps of acupuncture schedule, needling and needle remaining for 30 minutes.

Sham Acupuncture ${ }^{13}$ Protocol - the number, duration and frequency of the sham acupuncture group were consistent with those in the ATAS group. The sham protocol utilized fine and short needles for minimally invasive, shallow needles' insertion at non-acupuncture points. In each course of treatment, sixteen standardized points were needled bilaterally and superficially using fine needles (ie, superficial acupuncture at non-meridian points).

Acupuncture interventions were provided by licensed acupuncturists or medical doctor acupuncturists trained in the ATAS method. The acupuncturists were already on staff at the clinical sites or community-based clinics affiliated with the cancer center or academic medical center.

For description of ATAS and sham acupuncture, see

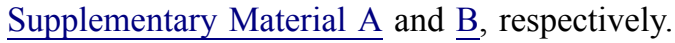

\section{Concomitant Treatments}

Patients were instructed not to take any additional medications during the study without prior consultation with the investigator. Medication to treat side effects like nausea, vomiting or diarrhea was allowed as per standards. G-CSF was allowed in the course of the study in the event of neutropenia or febrile neutropenia grade $\geq 3$.

\section{Diet and Physical Activity}

All participants received health education from nurses, telling them about their diet and precautions during 
chemotherapy, including eating high-quality protein such as fish and chicken, eating more fruits and vegetables, and not eating greasy, spicy and raw food. During hospitalization, the nutrition canteen helped deliver patients' meals.

All the participants were postoperative patients, nurses taught patients to do postoperative exercises step by step, helped patients with functional exercise. Nurses performed skin examination and functional recovery assessment before each chemotherapy session, and provided timely health education.

\section{Assessments}

Schedule of intervention and assessments (Figure 1):

longitudinal assessments of fatigue through VAS-F scale weekly during the whole course of chemotherapy and until 1 month after completion of chemotherapy; severe fatigue was defined as MFI-20 scores $\geq 60$ on dimension scales scored from 0 to $100 .^{14}$

Inventory Fatigue was assessed with the MFI-20 at baseline (T1), after 3-4 cycles of EC (T2), at the end of 3-4 cycles of paclitaxel (T3) and 1 month post-chemotherapy (T4). Insomnia is a common and debilitating disorder experienced by cancer survivors, acupuncture provides meaningful and durable improvement of insomnia. ${ }^{15}$ Insomnia is highly persistent, especially in female cancer patients. ${ }^{16}$ Insomnia has been associated with many clinical and psychological parameters, especially with fatigue. ${ }^{16}$ Sleep quality was assessed by the Insomnia Severity Index (ISI), which incorporates additional items on sleep quality and specific sleep-related daytime impairments (on daytime fatigue, cognitive functioning, mood, interpersonal relationship, and daily activities). ${ }^{17}$

\section{Data Collection}

For each patient included in the trial, a written informed consent had to be completed and signed by the investigator or the person designated by the investigator. The eCRF gathered both clinical data and party of the data collected during the analysis of samples. The data from the paper CRF were then recorded in an eCRF thanks to MACRO, software developed by InferMed. These input data were the data source for the statistical analysis.

\section{mRNA Sequencing}

The blood samples of all the participants were collected in Paxgene tubes at 4 timepointw as described previously, and stored in a refrigerator at -80 centigrade (which can make RNA stable at less 11 years).

After all the blood samples were collected, they were kept at room temperature for 2 hours, then put through centrifugal filtration. The RNA samples were extracted with Trizol. To ensure the good quality of samples for sequencing, qubit (Thermo, USA) was used to detect the concentration of the RNA sample. Then dscDNA synthesis and cluster Generation were performed. mRNA sequencing libraries were generated using the TruSeq strand RNA Prep Kit (illumine, USA) for Illumina (Nova6000, USA) according to the manufacturer's instructions.

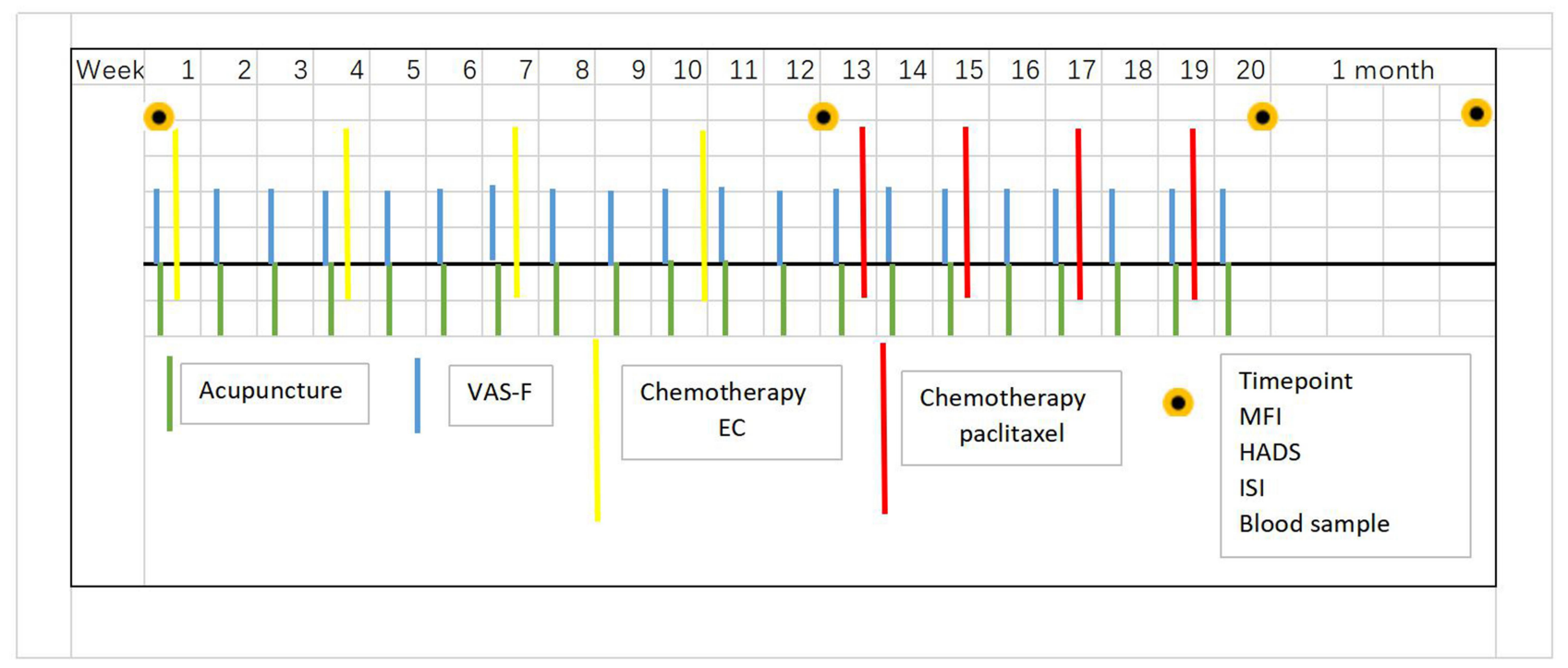

Figure I Timetable for acupuncture, chemotherapy and assessment. 


\section{Statistical Analysis}

The results are given as mean \pm SD for assessment scales. Variance analysis was used to test differences in three groups at baseline. Repeated measurement analysis of variance application was performed to analyze time effects and intergroup effects in the data of MFI-20, HADS and ISI. Differences were considered statistically significant at $\mathrm{P}<0.05$. Statistical analyses were conducted using SPSS.

\section{Results}

\section{Participants' Characteristics}

The pilot study in Kunming center started on May 3rd, 2018, recruitment ended on Nov 14th 2018, the last participant was followed up on May 10th, 2019. After screening for 258 patients, 40 patients were included in 6 months. 30 participants in acupuncture group (20 in ATAS, 10 in sham group, only acupuncture practitioner knows), and 10 in non-acupuncture group.

There were 3 patients who dropped out, 1 patient dropped out at the beginning, because they wanted to be in the acupuncture group, while randomized to nonacupuncture group; the other patient could not comply with the protocol and do the acupuncture once a week, and she only received 7 sessions of acupuncture (35\%) due to distance between her home and the hospital (more than 400 kilometers); the last patient dropped out after finishing 4 regimens of EC, discovering distant metastasis.

Good compliance was observed in this pilot study. A total of 581 sessions of acupuncture were performed on the patients in acupuncture group, mean acupuncture session was 20.03 for each person included in analyses, the number of which is in accordance with the requirement.

The mean age of participants was 47.5, 42 and 50.5 years old in ATAS, sham acupuncture and nonacupuncture group respectively. There was no difference between three groups in clinical characteristics (Table 1). There were three participants with concomitant disease, two patients had high blood pressure and one had type 2 diabetes. But the concomitant disease was stable - controlled by pills.

\section{Blinding and the Satisfaction of Acupuncture Treatment}

All of the patients in acupuncture group believed that they received true ATAS. The recruitment protocol was as shown in Figure 2.

\section{Outcomes}

There was no difference between three groups in mean (sd) scores of VAS-F, MFI-20, HADS and ISI at baseline (Table 2). So, it means that all the participants in different groups had similar levels of fatigue, depression, anxiety and sleep disorders at the time of randomizing to groups.

T4 is one month after the last cycle of chemotherapy. Some participants were receiving radiotherapy or endocrine therapy, this would probably influence the degree of fatigue. After discussion, we decided to abandon the $\mathrm{T} 4$ analysis in groups.

\section{VAS-F (Figure 3A)}

Every participant completed VAS-F each week via their smart phones according to their level of fatigue. The score of VAS-F ranges from 0 to 10 . The whole process was about 20 weeks, while some participants did not record the score of VAS-F after the 16th week, we analyzed the data from the first week to 16th week for some missing data after 17th week. The symptom scores of patients randomly assigned to the ATAS acupuncture group were significantly improved compared with the sham acupuncture and non-acupuncture groups since 7 weeks' treatment at VAS-F score, which was the time of starting the third cycle of chemotherapy. After that time, persistent ATAS acupuncture reduced fatigue significantly, even on the weekdays of receiving chemotherapy, while the other two groups experienced fluctuations during chemotherapy days. Compared with the sham acupuncture group, patients who were randomly assigned to ATAS acupuncture had improved symptoms throughout the course of chemotherapy. The VAS-F score of ATAS acupuncture decreased 0.351 and 1.198 compared with that of sham acupuncture and non-acupuncture groups. ATAS acupuncture vs non-acupuncture groups, $\mathrm{P}=0.004$.

\section{MFI-20 (Figure 3B)}

There was a sharp rise in score of MFI-20 in nonacupuncture and sham acupuncture groups since the chemotherapy started, which reached a peak of $56.8 \pm 18.7$ and $49.0 \pm 12.8$ score at T2, while the score of MFI- 20 of ATAS acupuncture group was well-controlled, slightly dropping from $46.4 \pm 9.1$ to $43.1 \pm 18.1$, which means that the fatigue could be treated by ATAS acupuncture during EC chemotherapy regiment. The period from $\mathrm{T} 2$ to $\mathrm{T} 3$ witnessed a decrease in non-acupuncture and sham acupuncture groups, along with the adverse effect of only one drug, 
Table I General Demographic and Disease Characteristics

\begin{tabular}{|c|c|c|c|c|}
\hline & & Group I & Group2 & Group3 \\
\hline & & $n=20$ & $n=10$ & $n=10$ \\
\hline Age & $\begin{array}{l}\text { Median age (range) } \\
30-39 \\
40-49 \\
50-59 \\
\text { Older than } 60\end{array}$ & $\begin{array}{l}47.5 \\
(40-62) \\
0 \\
12 \\
5 \\
3\end{array}$ & $\begin{array}{l}42 \\
(32-61) \\
3 \\
4 \\
2 \\
1\end{array}$ & $\begin{array}{l}50.5 \\
(33-70) \\
2 \\
2 \\
4 \\
2\end{array}$ \\
\hline Occupation & $\begin{array}{l}\text { Famer } \\
\text { Formal job } \\
\text { Jobless }\end{array}$ & $\begin{array}{l}6 \\
7 \\
7\end{array}$ & $\begin{array}{l}2 \\
7 \\
1\end{array}$ & $\begin{array}{l}2 \\
6 \\
2\end{array}$ \\
\hline BMI & $\begin{array}{l}<20 \\
20-23.9 \\
\geq 24.0\end{array}$ & $\begin{array}{l}3 \\
11 \\
6\end{array}$ & $\begin{array}{l}3 \\
7 \\
0\end{array}$ & $\begin{array}{l}2 \\
4 \\
4\end{array}$ \\
\hline Staging & $\begin{array}{l}\text { I a } \\
\text { II a } \\
\text { II b } \\
\text { III a } \\
\text { III c }\end{array}$ & $\begin{array}{l}5 \\
7 \\
2 \\
5 \\
1\end{array}$ & $\begin{array}{l}1 \\
6 \\
1 \\
2 \\
0\end{array}$ & $\begin{array}{l}0 \\
3 \\
3 \\
3 \\
1\end{array}$ \\
\hline $\begin{array}{l}\text { Pathological } \\
\text { grading }\end{array}$ & $\begin{array}{l}\text { Poorly differentiated } \\
\text { Moderately } \\
\text { differentiated }\end{array}$ & $\begin{array}{l}4 \\
16\end{array}$ & $\begin{array}{l}1 \\
9\end{array}$ & $\begin{array}{l}1 \\
9\end{array}$ \\
\hline $\begin{array}{l}\text { Molecular } \\
\text { subtype }\end{array}$ & $\begin{array}{l}\text { Luminal A } \\
\text { Luminal B Her2 } \\
\text { positive subtype } \\
\text { Luminal B Her2 } \\
\text { negative subtype } \\
\text { Triple negative }\end{array}$ & $\begin{array}{l}5 \\
7 \\
7 \\
1\end{array}$ & $\begin{array}{l}2 \\
5 \\
2 \\
1\end{array}$ & $\begin{array}{l}4 \\
2 \\
3 \\
1\end{array}$ \\
\hline $\begin{array}{l}\text { Operation } \\
\text { type }\end{array}$ & $\begin{array}{l}\text { Breast-conserving } \\
\text { surgery } \\
\text { Modified radical } \\
\text { surgery }\end{array}$ & $\begin{array}{l}5 \\
15\end{array}$ & $\begin{array}{l}2 \\
8\end{array}$ & $\begin{array}{l}2 \\
8\end{array}$ \\
\hline $\begin{array}{l}\text { Concomitant } \\
\text { disease }\end{array}$ & $\begin{array}{l}\text { No } \\
\text { Yes }\end{array}$ & $\begin{array}{l}18 \\
2\end{array}$ & $\begin{array}{l}9 \\
1\end{array}$ & $\begin{array}{l}10 \\
0\end{array}$ \\
\hline
\end{tabular}

paclitaxel, which was less than that of two drugs, EC chemotherapy regimen. The score of MFI-20 in ATAS acupuncture group was almost maintained, for it had already been a very low score. Therefore, we can estimate that ATAS acupuncture can decrease chemotherapy induced fatigue. Though the sample was very small, there was still a statistical difference between ATAS and non-acupuncture, sham and non-acupuncture, $\mathrm{P}=0.016$, 0.028 respectively.

\section{HADS (Figure 3C)}

It was obvious that HADS score had been dramatically decreasing in the whole process in the ATAS acupuncture group while the score was almost maintained in nonacupuncture group. There was a fluctuation in data of sham-acupuncture group, with climbing, and then dropping. The score in the ATAS acupuncture group decreased by 0.513 and 1.893 compared with that of sham and nonacupuncture groups. When comparing sham and nonacupuncture groups, the score in sham group decreased by 1.380 . Unfortunately, there was no statistical difference since the sample was not big enough to reach a positive result. But we can still conclude that ATAS acupuncture can alleviate depression and anxiety with an obvious trend.

\section{ISI (Figure 3D)}

The score of Insomnia Severity Index had a similar trend in three groups during the period T1 to T2, with a sharp rise in sham and non-acupuncture groups. Then it decreased in ATAS and non-acupuncture groups. The score of the patients in ATAS acupuncture group keep bottom from T2 to T3. The score in the ATAS acupuncture group decreased by 0.874 and 0.630 compared with that of sham and non-acupuncture groups.

\section{Adverse Effects}

There were no severe adverse effects induced by acupuncture, only when participants did not properly press acupoints after the acupuncture, they experienced bluish bruising, which would resolve by itself after 1-2 days.

\section{mRNA Sequencing Outcomes}

There were 146 blood samples collected in total from participants at 4 timepoints. Though some of the blood samples did not meet the required concentration, tubes b compensated. Because 2 tubes of blood were collected at each timepoint from all the participants.

According to the mRNA sequencing, there were 56 differential genes and 6 significantly enriched genes in ATAS and non-acupuncture groups. Enriched genes were ADORA1, KCNMA1, DEFA3, ELANE, PAX8, CTSG (Figure 4). These six differential genes were searched and their functions were analyzed in PubMed. The main gene related to fatigue and depression was ADORA1, which was closely related to cGMP/PKG signaling pathway (Figure 5). ADORA1 was upstream of cGMP/PKG signaling pathway in KEEG.

\section{Discussion}

In this pilot study, sham and non-acupuncture groups of patients with stage I-III breast cancer receiving EC-T 


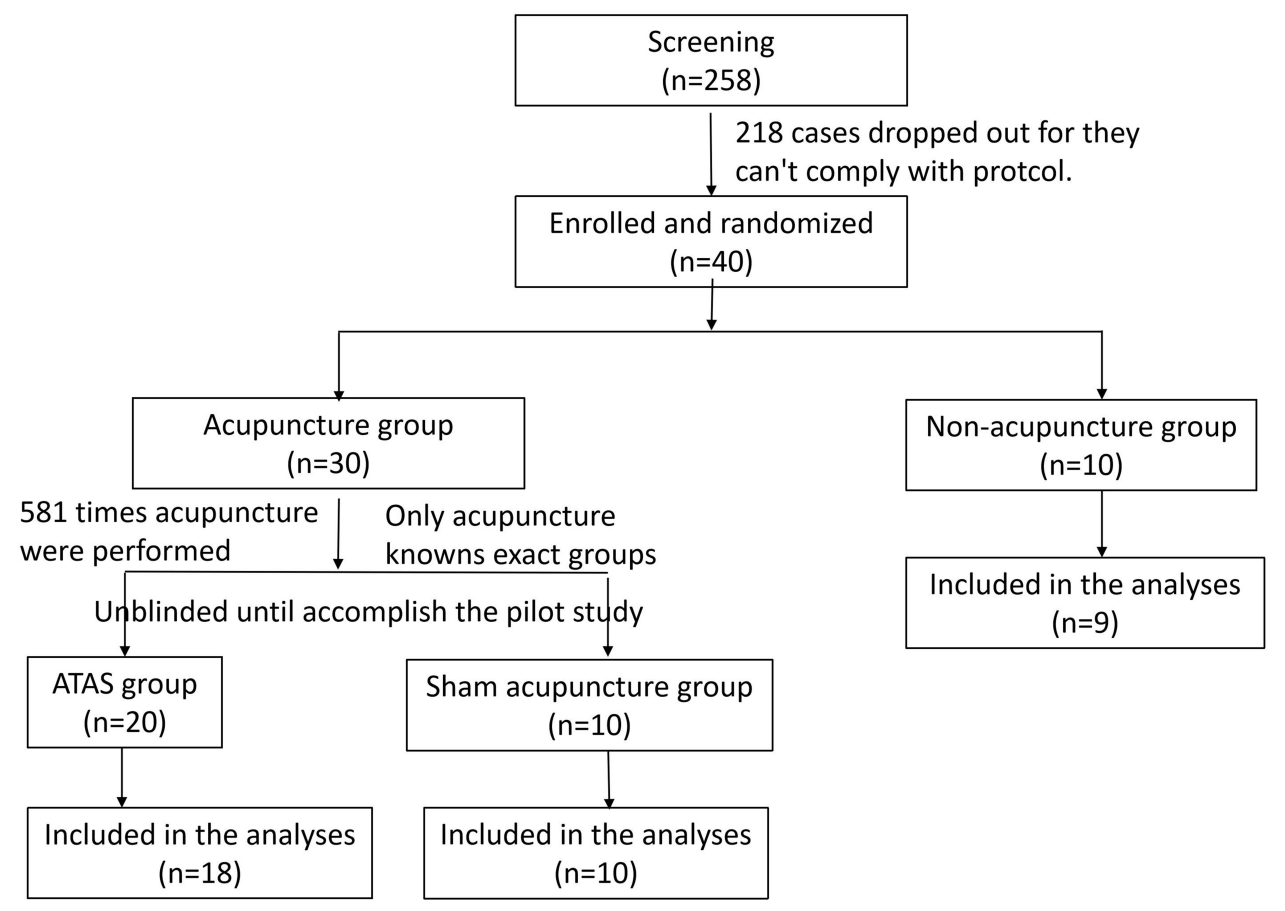

Figure 2 Diagram of screening, enrollment, randomization and follow-up.

chemotherapy experienced high levels of fatigue, depression and insomnia. There were statistically significant improvements in fatigue scores with ATAS acupuncture administered once a week for 20 weeks compared with both sham and non-acupuncture. Patients randomized to ATAS acupuncture had improved symptoms during the whole process of chemotherapy compared with the sham acupuncture group. We estimate that the between-group difference will appear with larger sample.

In oncology practice, Chinese medicine is commonly used to reduce side-effects from chemotherapy or radiation therapy, to aid recovery after an operation, to palliate symptoms and to address survivorship issues. ${ }^{18}$ ASCO Endorsement of the SIO Clinical Practice Guideline indicated that acupressure and acupuncture are recommended for reducing chemotherapy-induced nausea and vomiting. ${ }^{19,20}$ Currently, there are a lot of trials that have reported that acupuncture treats cancer patients in order to palliate symptoms. A survey showed that $61 \%$ of cancer patients experienced pain, $74 \%$ of cancer patients experienced fatigue, and $46 \%$ of cancer patients experienced distress. ${ }^{21}$ Hence, targeting women at risk for severe fatigue is essential to increase the life quality of breast cancer patients. A study which characterized cyclical variations in fatigue across a taxane-based chemotherapy cycle showed that fatigue increased from baseline to prior to the third treatment, then peaked at 3-5 days after the third treatment, before recovering prior to the next treatment. ${ }^{22}$ Our result was similar to this, through VAS-F, we can see that the scores of VAS-F were climbing at the time of starting the third cycle chemotherapy in sham and nonacupuncture groups.

In the study, 146 blood samples were collected from participants, mRNA sequencing was performed to explore the mechanism through which acupuncture reduces chemotherapy induced fatigue. The occurrence of fatigue involves many factors such as physiology, biochemistry, psychology, spirit and biological rhythm. It is closely

Table 2 Baseline Mean Scores in Different Groups

\begin{tabular}{|l|l|l|l|l|l|}
\hline & ATAS Acupuncture & Sham Acupuncture & Non-Acupuncture & F & P \\
\hline VAS-F & $1.3 \pm 1.2$ & $1.3 \pm 1.0$ & $2.1 \pm 1.5$ & 1.547 & 0.229 \\
MFI-20 & $46.4 \pm 9.1$ & $39.3 \pm 12.5$ & $45.8 \pm 15.7$ & 1.031 & 0.368 \\
HADS & $10.2 \pm 6.1$ & $8.3 \pm 6.1$ & $9.4 \pm 3.9$ & 1.072 & 0.354 \\
ISI & $4.5 \pm 3.5$ & $3.0 \pm 3.7$ & $5.6 \pm 4.4$ & 0.739 & 0.485 \\
\hline
\end{tabular}



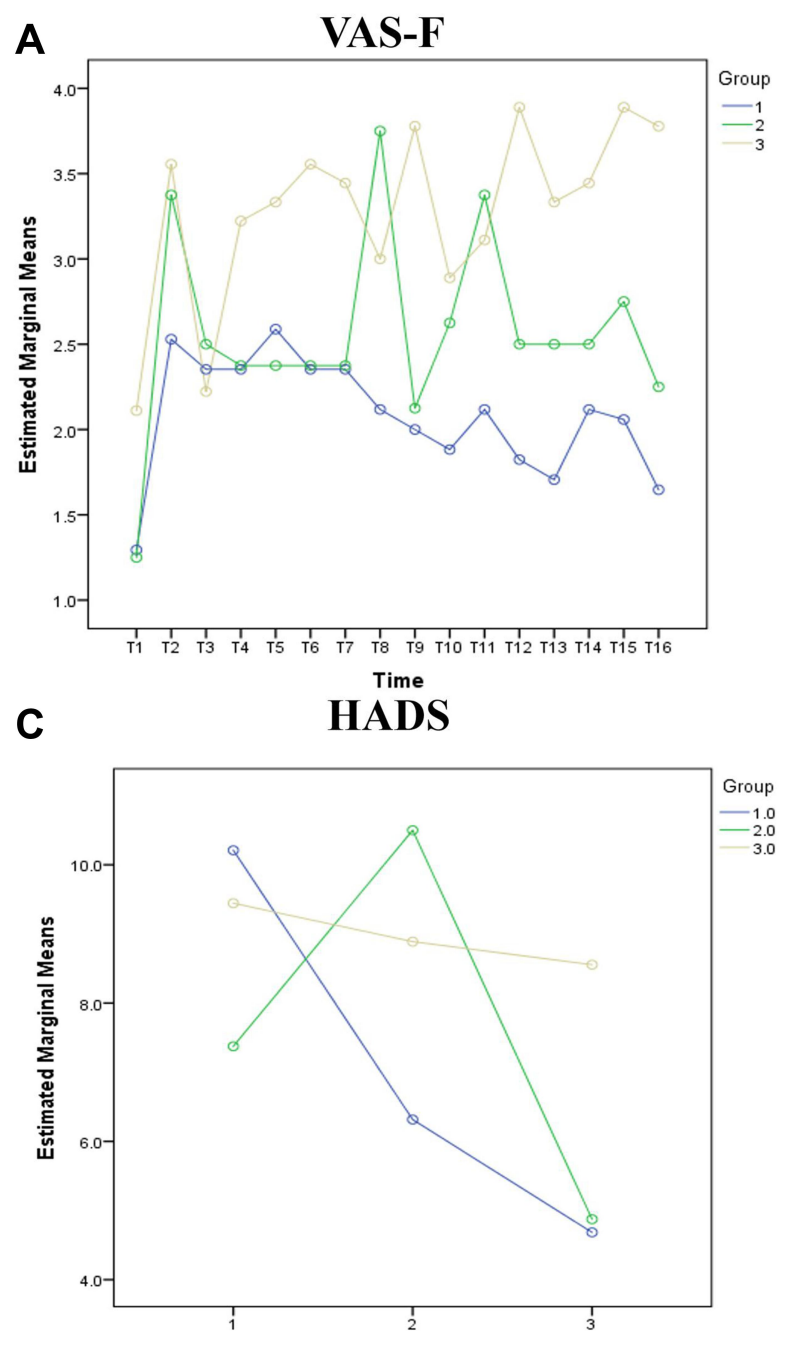

B

MFI-20

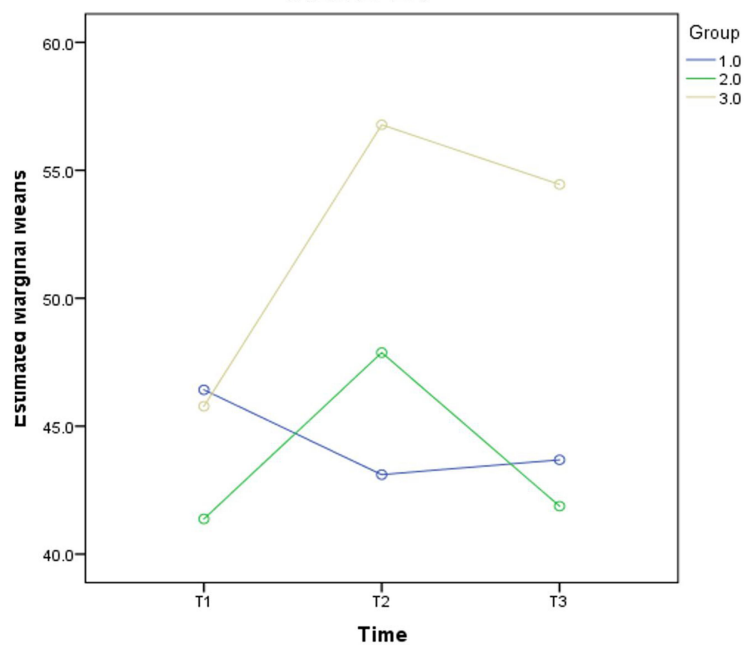

D

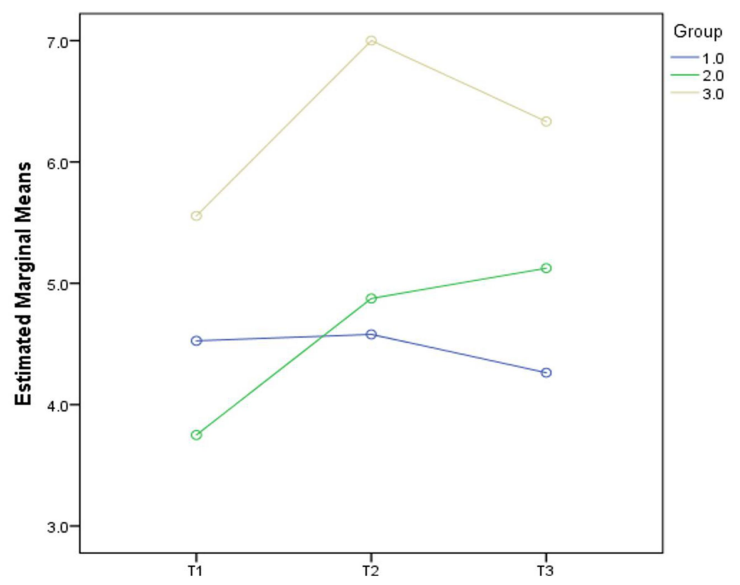

Figure 3 The mean score of assessment scales at different timepoints in three groups (*Group I: ATAS acupuncture group; Group 2: sham acupuncture group; Group 3: non-acupuncture group). (A) VAS-F, (B) MFI-20, (C) HADS, (D) ISI.

related to the dysfunction of the internal neuroendocrine immune network. Depression is one of the symptoms of cancer-related fatigue, higher C-reactive protein levels were significantly associated with pre chemotherapy fatigue and depression. ${ }^{23}$ Some studies showed that acupuncture and moxibustion treatment of cancer-related fatigue mainly changes the immune status of the body by regulating the HPA axis, and the cause of HPA axis change is not clear, which may be related to multiple signaling pathways. $^{24}$ Acupuncture can effectively reduce the expression of NF- $\kappa \mathrm{B}$, the level of COX-2 and PGE2 in the hippocampus of depressed mice, which may play an antidepressant role by inhibiting the pro-inflammatory pathway and protecting the neurons in the hippocampus. ${ }^{25}$

ADORA1 is a subtype of adenosine receptor related to cell death. Long-term exposure to environmental pressure will lead to hypothalamic pituitary adrenal (HPA) axis disorder and oxidative stress, which is related to the development of depression like behavior. ADORA1 is involved in down regulating inflammatory factors and promoting smooth muscle cell proliferation. ${ }^{26}$ After long-term caffeine treatment, combined with caffeine and gossypol or agomelatonin, ADORA1 was involved in an antidepressant like effect, ${ }^{27}$ and caffeine withdrawal resulted in a decrease in the level of ADORA1 mRNA in the cerebral cortex. $^{28}$ Through the mechanism of no/cGMP/PKG/ KATP intracellular signaling pathway, peripheral activation of ADORA1 reduces inflammatory pain sensation. ${ }^{29}$ CGMP/PKG signaling pathway plays a key role in regulating cell growth, metabolism and many other intracellular processes. The role of $\mathrm{cGMP} / \mathrm{PKG}$ signaling in neuropathic and inflammatory pain has also been observed in many other animal models, and it involves many mechanisms of ACTH. ${ }^{30}$ Through the no/cGMP/PKG/ 


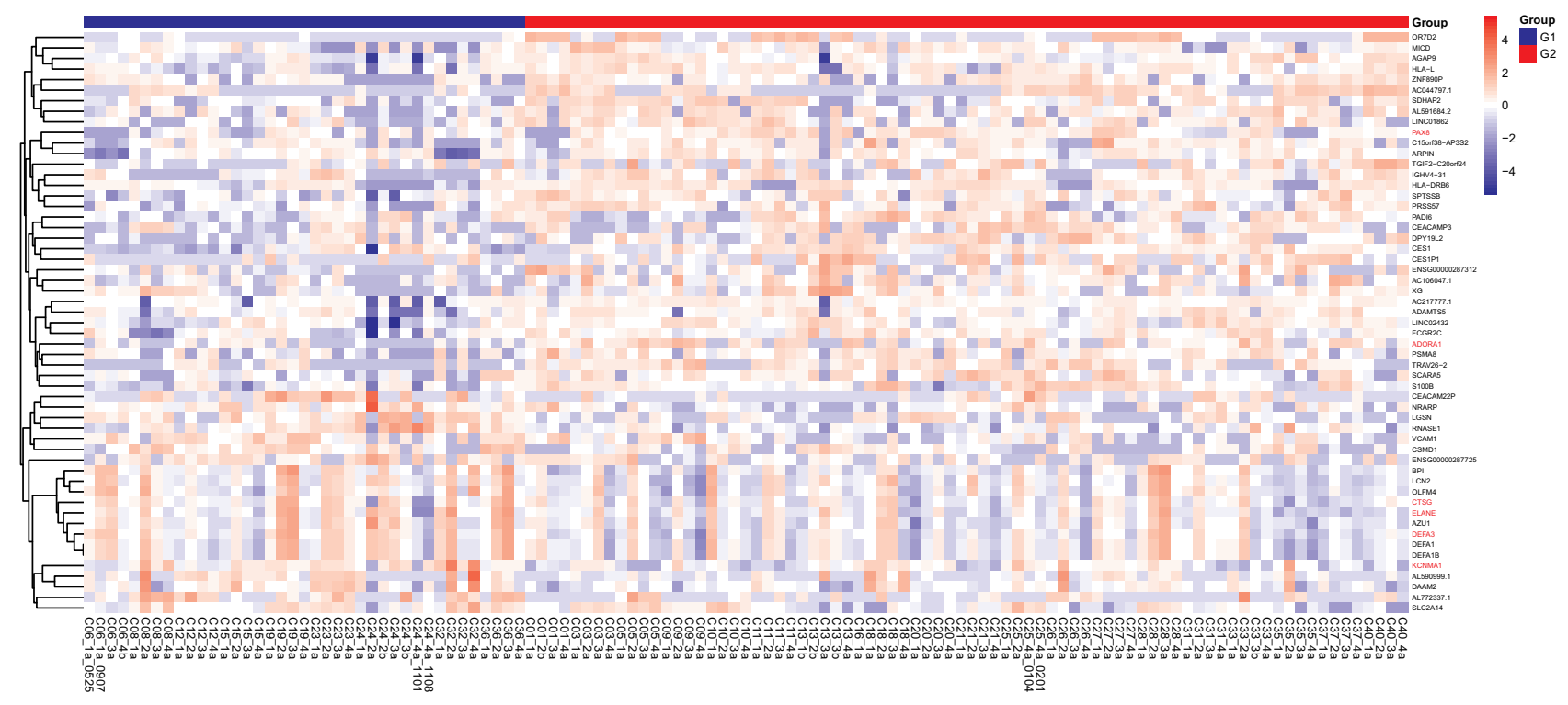

Figure 4 Differential mRNA clustering thermogram in ATAS and non-acupuncture groups (GI: non-acupuncture group, G2: ATAS group).
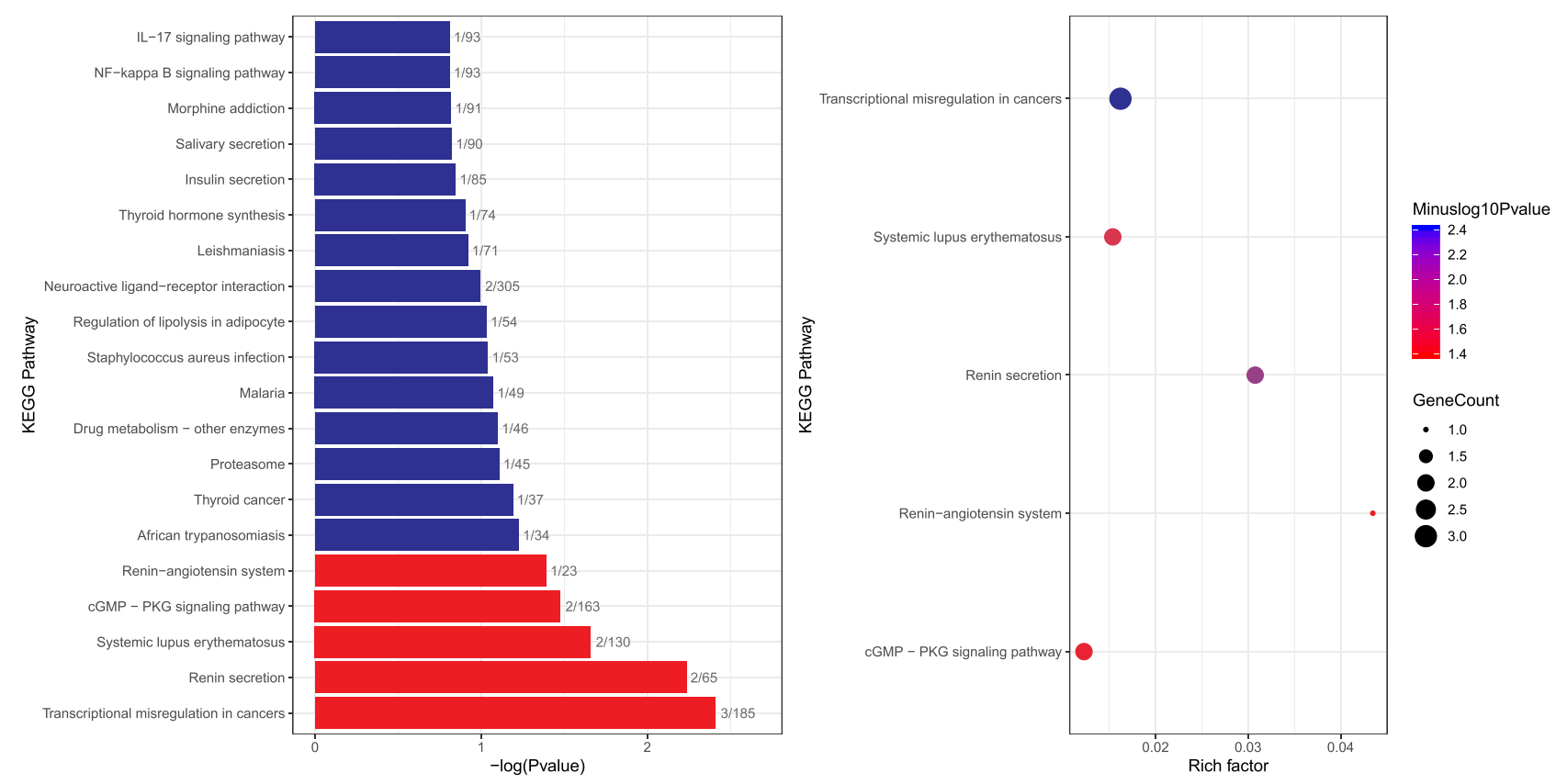

Figure 5 Significantly enriched gene pathway.

KATP intracellular signaling pathway, peripheral activation of ADORA1 reduces inflammatory pain perception. ${ }^{29}$

The expression of ADORA1 is related to HPA axis maladjustment, which is involved in down-regulation of inflammatory factors; cGMP/PKG signaling pathway plays an important role in regulating cell metabolism, including proinflammatory factors and adrenocorticotropic hormone mechanism; in addition, peripheral activation of ADORA1 can regulate cGMP/PKG signaling pathway, participate in regulation of proinflammatory factors and HPA axis, and regulate antidepressant and anti-anxiety like behaviors. The clinical study found that the symptoms of fatigue and depression were alleviated after the treatment of breast cancer by acupuncture and moxibustion. We speculate that acupuncture may activate ADORA1 in vivo, regulate cGMP/PKG signaling pathway, then reduce fatigue and depression in breast cancer patients (Figure 6). More investigations are required to explore the mechanism of acupuncture. 


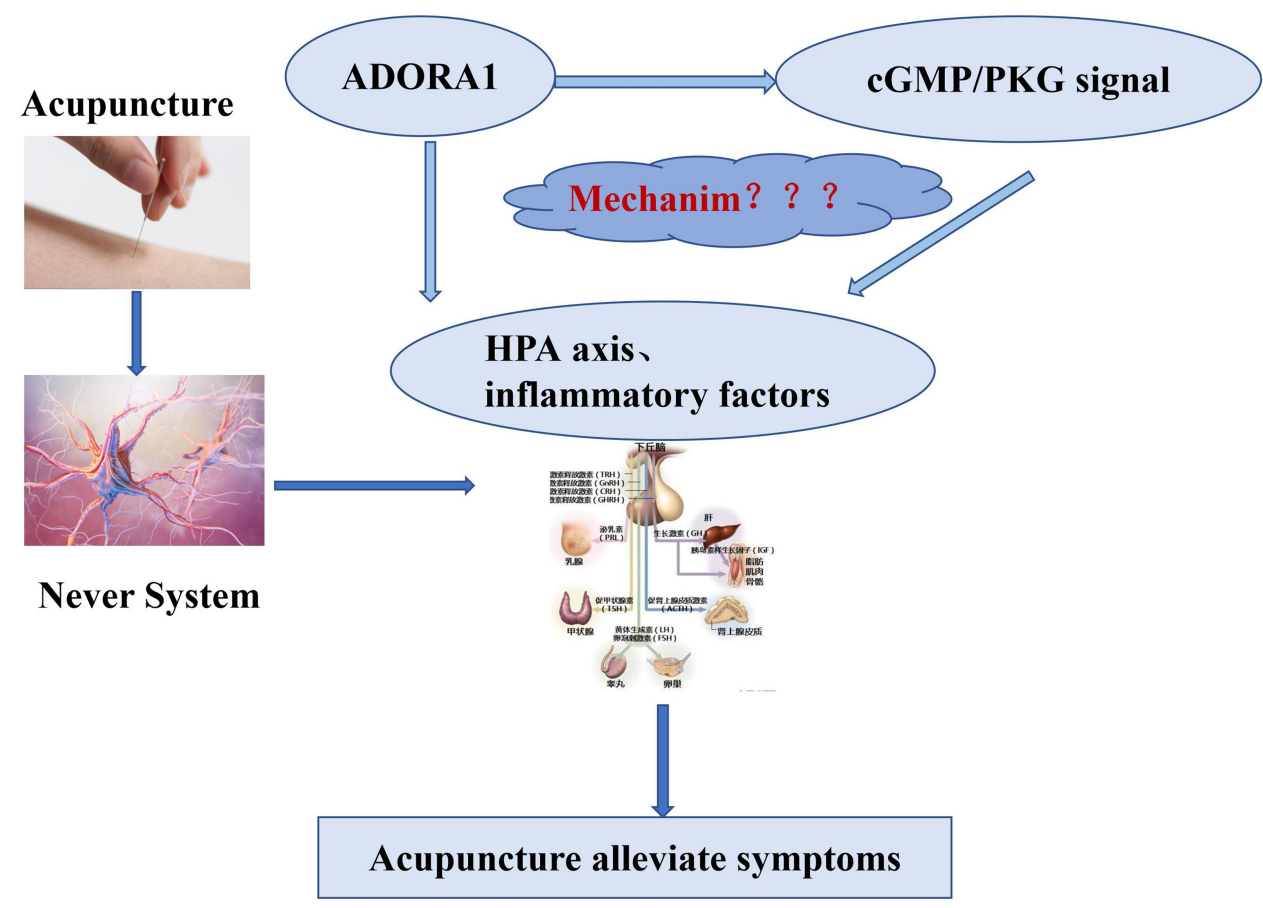

Figure 6 Hypothesis of mechanism.

This study has intervention was strictly conducted following the protocol which underwent standardized training and administered the repeat assessments throughout the time frame of the study. All the participants received the same chemotherapy regimens ranging 5 months. Acupuncturists had the same training backgrounds, which would secure the homogeneity of acupuncture. Smart phones were used to collect data for doctors and patients, which can automatically provide assessment scales to participants. There were similar retention and completion rates for each group, thereby reducing the concerns about differential dropout. mRNA sequencing was performed trying to explore the mechanism of acupuncture alleviating symptoms.

\section{Conclusion}

1) ATAS acupuncture can significantly reduce fatigue, maintain a stable low level of fatigue during 8 cycles of chemotherapy.

2) ATAS acupuncture can decrease depression, anxiety and insomnia induced by chemotherapy.

3) Acupuncture may activate ADORA1, regulate cGMP/PKG signaling pathway, then reduce fatigue and depression in breast cancer patients.

\section{Data Sharing Statement}

Yes, we intend to share individual de-identified participant data. The data were recorded in eCRF, and exported by Excel. We can provide data if requested. Please contact Yun-fen LI (408,140,057@qq.com) if you need the data.

\section{Ethics Approval and Consent to Participate}

We confirm that this trial was conducted in accordance with the Declaration of Helsinki. The study was approved by the ethics committee of Yunnan cancer hospital (YJZ 201,705; Supplementary material C).

\section{Consent for Publication}

All the authors agreed to the submission.

\section{Acknowledgments}

We are grateful to the support of all the specialists who were involved in the discussion of the study protocol.

We must acknowledge all the clinicians, nurses and acupuncturists who participated in clinical research implementation.

Yong ZHANG, Shao-Qiang ZHOU, Chun-Xiang LI and Chang-An Wang are clinicians, responsible for the 
strategy of anti-cancer treatment and recording of the data. Li-Juan LIU, Qiong MA and Rui-Ming MA are research nurses, responsible for reminding patients to follow the procedure to answer the questionnaires. Mei HUANG, Rang SONG, Xiong-Ying BAO, Ling-Ling WANG and Ying-Hao WANG are acupuncturists, responsible for doing ATAS and sham acupuncture for participants.

\section{Author Contributions}

All authors made substantial contributions to conception and design, acquisition of data, or analysis and interpretation of data; took part in drafting the article or revising it critically for important intellectual content; agreed on the journal to which the article will be submitted; gave final approval of the version to be published; and agree to be accountable for all aspects of the work.

Mian-Sheng Zhu and Wen-Hui LI are co-corresponding authors, Yun-Fen LI, De-Quan LIU, Jian-Yun NIE and DeDian CHEN contributed equally.

\section{Funding}

This trial (ChiCTR-IPR-17013652) is granted by the Key research and development project of Yunnan provincial science and technology department (2018IA059) (for further details see Supplementary material D), governmental funding. The funding agencies were not involved in the design and conduct of the study, nor in the collection, management, analysis, and interpretation of the data. They were not involved in the writing of the manuscript.

\section{Disclosure}

Zheng Zuo reports that the ATAS acupuncture technology belongs to the acupuncture team. Wei-Yu Wan is an employee of Servbus Technology (Beijing) Co., Ltd. The authors report no other potential conflicts of interest for this work.

\section{References}

1. Campos MP, Hassan BJ, Riechelmann R, et al. Cancer-related fatigue: a practical review. Ann Oncol. 2011;22(6):1273-1279. doi:10.1093/ annonc/mdq 458

2. Bower JE, Ganz PA, Desmond KA, et al. Fatigue in breast cancer survivors: occurrence, correlates, and impact on quality of life. J Clin Oncol. 2000;18(4):743-753. doi:10.1200/JCO.2000.18.4.743

3. Goldstein D, Bennett BK, Webber K, et al. Cancer-related fatigue in women with breast cancer: outcomes of a 5-year prospective cohort study. J Clin Oncol. 2012;30(15):1805-1812. doi:10.1200/ JCO.2011.34.6148

4. Parkin DM, Fernandez LMG. Use of statistics to assess the global burden of breast cancer. Breast J. 2006;12(Suppl 1):S70-80. doi:10.1111/j.1075-122X.2006.00205.x
5. Lehmann H. Acupuncture in ancient China: how important was it really? J Integr Med. 2013;11(1):45-53. doi:10.3736/jintegrmed2013008

6. Deng G, Cassileth BR, Yeung KS. Complementary therapies for cancer-related symptoms. J Support Oncol. 2004;2(5):419-426.

7. Molassiotis A, Bardy J, Finnegan-John J, et al. Acupuncture for cancer-related fatigue in patients with breast cancer: a pragmatic randomized control led trial. J Clin Oncol. 2012;30(36):4470-4476. doi:10.1200/JCO.2012.41.6222

8. Mao H, Mao JJ, Guo M, et al. Effects of infrared laser moxibustion on cancer-related fatigue: a randomized, double-blind, placebo- controlled trial. Cancer. 2016;122(23):3667-3672. doi:10.1002/ cncr.30189

9. Mao JJ, Farrar JT, Bruner D, et al. Electroacupuncture for fatigue, sleep, and psychological distress in breast cancer patients with arom atase inhibitor-related arthralgia: a randomized trial. Cancer. 2014;120(23):3744-3751. doi:10.1002/cncr.28917

10. Molassiotis A, Bardy J, Finnegan-John J, et al. A randomized, controlled trial of acupuncture self-needling as maintenance therapy for cancer-related fatigue after therapist-delivered acupuncture. Ann Oncol. 2013;24(6):1645-1652. doi:10.1093/annonc/mdt034

11. Hampton T. Cancer investigators make strides in improving patients' quality of life. JAMA. 2008;300(4):383.

12. Vickers AJ, Straus DJ, Fearon B, et al. Acupuncture for postchemotherapy fatigue: a Phase II study. J Clin Oncol. 2004;22 (9):1731-1735. doi:10.1200/JCO.2004.04.102

13. Azad A, John T. Do randomized acupuncture studies in patients with cancer need a sham acupuncture control arm? J Clin Oncol. 2013;31 (16):2057-2058. doi:10.1200/JCO.2012.47.8750

14. Mounier N, Anthony S, Busson R, et al. Long-term fatigue in survivors of non-Hodgkin lymphoma: the lymphoma study association SIMONAL cross- sectional study. Cancer. 2019.

15. Garland SN, Xie SX, DuHamel K, et al. Acupuncture versus cognitive behavioral therapy for insomnia in cancer survivors: a randomized clinic al trial. J Natl Cancer Inst. 2019;111 (12):1323-1331. doi:10.1093/jnci/djz050

16. Schieber K, Niecke A, Geiser F, et al. The course of cancer-related insomnia: don't expect it to disappear after cancer treatment. Sleep Med. 2019;58:107-113. doi:10.1016/j.sleep.2019.02.018

17. Ji X, Ivers H, Savard J, et al. Residual symptoms after natural remission of insomnia: associations with relapse over 4 years. Sleep. 2019;42(8). doi:10.1093/sleep/zsz122.

18. So TH, Chan SK, Lee VH, et al. Chinese medicine in cancer treatment - how is it practised in the east and the west? Clin Oncol. 2019;31(8):578-588. doi:10.1016/j.clon.2019.05.016

19. Lyman GH, Greenlee H, Bohlke K, et al. Integrative therapies during and after breast cancer treatment: ASCO endorsement of the SIO clinical practice guideline. J Clin Oncol. 2018;36(25):2647-2655. doi:10.1200/JCO.2018.79.2721

20. Greenlee H, DuPont-Reyes MJ, Balneaves LG, et al. Clinical practice guidelines on the evidence-based use of integrative therapies during and after brea st cancer treatment. CA Cancer J Clin. 2017;67 (3):194-232.

21. Smith TG, Troeschel AN, Castro KM, et al. Perceptions of patients with breast and colon cancer of the management of cancer-related pain, fatigu e, and emotional distress in community oncology. J Clin Oncol. 2019; Jco1801579.

22. Kirkham AA, Bland KA, Zucker DS, et al. "Chemotherapyperiodized" exercise to accommodate for cyclical variation in fatigue. Med Sci Sports Exerc. 2019.

23. Pertl MM, Hevey D, Boyle NT, et al. C-reactive protein predicts fatigue independently of depression in breast cancer patients prior to chemotherapy. Brain Behav Immun. 2013;34:108-119. doi:10.1016/j. bbi.2013.07.177

24. Yang S, Chu S, Gao Y, et al. A narrative review of cancer-related fatigue (CRF) and its possible pathogenesis. Cells. 2019;8(7):738. doi:10.3390/cells 8070738 
25. Shao RH, Jin SY, Lu J, et al. Effect of acupuncture intervention on expression of NF-| $\hat{E} B$ signal pathway in the hippocampus of chronic stress-induced depression rats. Zhen Ci Yan Jiu. 2015;40(5):368-372.

26. Simard T, Jung R, Labinaz A, et al. Adenosine as a marker and mediator of cardiovascular homeostasis: a translational perspective. Cardiovasc Hematol Disord Drug Targets. 2019;19(2):109-131.

27. Szopa A, Poleszak E, Doboszewska U, et al. Withdrawal of caffeine after its chronic administration modifies the antidepressant-like activity of atypical antidepressants in mice. Changes in cortical expression of Comt, Slc6a15 and Adora1 genes. Psychopharmacology (Berl). 2018;235(8):2423-2434. doi:10.1007/s00213-018-4940-6

28. Szopa A, Doboszewska U, Herbet M, et al. Chronic treatment with caffeine and its withdrawal modify the antidepressant-like activity of selecti ve serotonin reuptake inhibitors in the forced swim and tail suspension tests in mice. Effects on Comt, Slc6a15 and Adora1 gene expression. Toxicol Appl Pharmacol. 2017;337::95-103. doi:10.1016/ j.taap.2017.10.020

29. Lima FO, Souza GR, Verri WA, et al. Direct blockade of inflammatory hypernociception by peripheral A1 adenosine receptors: involvement of the NO/cGMP/PKG/KATP signaling pathway. Pain. 2010;151(2):506-515. doi:10.1016/j.pain.2010.08.014
30. Li ZH, Cui D, Qiu CJ, et al. Cyclic nucleotide signaling in sensory neuron hyperexcitability and chronic pain after nerve injury. Neurobiol Pain. 2019;6:100028. doi:10.1016/j.ynpai.2019.100028

31. Lee KA, Hicks G, Nino-Murcia G. Validity and reliability of a scale to assess fatigue. Psychiatry Research. 1991;(36);291-298

32. Smets EMA, Garssen B, Bonke B, Dehaes JCJM. The Multidimensional Fatigue Inventory (MFI): Psychometric qualities of an instrument to assess fatigue. J Psychosom Res. 1995;39;315-325

33. Bjelland I, Dahl AA, Haug TT, et al. The validity of the hospital anxiety and depression scale. An updated literature review. $J$ Psychosom Res. 2002;52:69-71

34. Bastien $\mathrm{CH}$, Vallieres A, Morin CM. Validation of the Insomnia Severity Index as an outcome measure for insomnia research. Sleep Med. 2001;2:297-307

\section{Publish your work in this journal}

OncoTargets and Therapy is an international, peer-reviewed, open access journal focusing on the pathological basis of all cancers, potential targets for therapy and treatment protocols employed to improve the management of cancer patients. The journal also focuses on the impact of management programs and new therapeutic agents and protocols on patient perspectives such as quality of life, adherence and satisfaction. The manuscript management system is completely online and includes a very quick and fair peer-review system, which is all easy to use. Visit http://www.dovepress.com/ testimonials.php to read real quotes from published authors. 